\title{
¿How Do Ocean Warm Anomalies Favor the Aggregation of Deep Convective Clouds?
}

\author{
Sara Shamekh, Caroline Muller, Jean-Philippe Duvel, and Fabio D'Andrea \\ Laboratoire de Météorologie Dynamique/IPSL, École Normale Supérieure, PSL Research University, CNRS, Paris, France
}

(Manuscript received 20 December 2018, in final form 29 May 2019)

\begin{abstract}
We investigate the role of a warm sea surface temperature (SST) anomaly (hot spot of typically 3 to $5 \mathrm{~K}$ ) on the aggregation of convection using cloud-resolving simulations in a nonrotating framework. It is well known that SST gradients can spatially organize convection. Even with uniform SST, the spontaneous self-aggregation of convection is possible above a critical SST (here $295 \mathrm{~K}$ ), arising mainly from radiative feedbacks. We investigate how a circular hot spot helps organize convection, and how self-aggregation feedbacks modulate this organization. The hot spot significantly accelerates aggregation, particularly for warmer/larger hot spots, and extends the range of SSTs for which aggregation occurs; however, at cold SST $(290 \mathrm{~K})$ the aggregated cluster disaggregates if we remove the hot spot. A large convective instability over the hot spot leads to stronger convection and generates a large-scale circulation which forces the subsidence drying outside the hot spot. Indeed, convection over the hot spot brings the atmosphere toward a warmer temperature. The warmer temperatures are imprinted over the whole domain by gravity waves and subsidence warming. The initial transient warming and concomitant subsidence drying suppress convection outside the hot spot, thus driving the aggregation. The hotspot-induced large-scale circulation can enforce the aggregation even without radiative feedbacks for hot spots sufficiently large/warm. The strength of the large-scale circulation, which defines the speed of aggregation, is a function of the hot spot fractional area. At equilibrium, once the aggregation is well established, the moist convective region with upward midtropospheric motion, centered over the hot spot, has an area surprisingly independent of the hot spot size.
\end{abstract}

KEYWORDS: Atmospheric circulation; Convection; Feedback; Radiative-convective equilibrium; Sea surface temperature; Cloud resolving models

\section{Introduction}

In the tropics, convection can be organized by synoptic dynamical systems such as equatorial waves or tropical depressions, but it may also have its own organization sources such as in squall lines, or more generally in mesoscale convective systems. Organized convection is associated with extreme weather conditions (Houze 2004), and can strongly impact the hydrological cycle and the top-of-atmosphere radiation budget (Tan et al. 2015; Tobin et al. 2012). For large-scale processes such as the Madden Julian oscillation, the aggregation of the convection may generate nonlinear effects modifying the average circulation at basin scale (Bellenger et al. 2009). However, the physical processes responsible for the mesoscale organization of convection are still not clearly identified and are typically not specifically accounted for in global climate models (GCMs) (Mapes and Neale 2011).

The spontaneous clustering of convective clouds in simulations in idealized settings, typically nonrotating radiative-convective equilibrium (RCE), provides a manageable framework to gain fundamental understanding of the physical processes at stake. Nonrotating RCE is an idealization of the tropical atmosphere where Earth's rotation is neglected, a reasonable approximation in the deep tropics where the Coriolis parameter is small, and where the large-scale circulation (larger than the model domain) is neglected. In other words, in RCE, there is no

¿ Denotes content that is immediately available upon publication as open access.

Corresponding author: Sara Shamekh, shamekh@lmd.ens.fr advection of energy into or out of the domain. Thus in the domain mean, surface latent and sensible heat fluxes are in balance with the net radiative cooling of the atmosphere (top-ofatmosphere minus surface).

In the tropics, such equilibrium is only reached at large, thousands of kilometers scales (Muller and O'Gorman 2011). The idealized framework of RCE has proven to be useful to study and improve our understanding of numerous aspects of tropical convection, including precipitation extremes (Muller et al. 2011; Muller 2013), entrainment (Romps 2010), cold pools (Tompkins 2001a), atmospheric thermodynamics (Pauluis and Held 2002) or rain evaporation (Muller and Bony 2015). Notably, it has led to the discovery of the remarkable ability of deep convection to spontaneously cluster in space despite homogeneous forcing in cloud-resolving models (CRMs). These are models with sufficient kilometer-scale horizontal resolutions to resolve the main features of deep convection, instead of parameterizing them.

Typical RCE simulations with homogeneous forcing [doubly periodic geometry, square domain, constant sea surface temperature (SST) in space and time] reach a statistically steady state in which convection and clouds are somewhat randomly distributed. But under certain conditions, including large domains, deep clouds aggregate into a region of the domain, surrounded by a dry environment devoid of deep convection. This phenomenon, known as self-aggregation in the literature [see, e.g., Wing et al. (2017) for a review], leads to an

Publisher's Note: This article was revised on 9 November 2020 to designate it as open access. 
equilibrium state with dry and warm mean thermodynamic profiles, and enhanced outgoing longwave radiation (OLR) to space (Bretherton et al. 2005; Tobin et al. 2012). Since its discovery in idealized CRM simulations, the self-aggregation of deep convection has been confirmed to occur in more realistic settings (Holloway 2017) and even in GCMs with parameterized convection (Coppin and Bony 2015).

Radiative feedbacks are believed to be key for self-aggregation, at least at temperatures observed in the tropical atmosphere (Wing et al.2017). It is the circulation generated by the differential longwave radiative cooling rates between dry (strong cooling) and moist (little cooling or even warming) regions which is believed to trigger and maintain the convective aggregation (Bretherton et al. 2005; Muller and Held 2012). Strong cooling in dry regions yields subsidence down to low levels, and a near-surface flow from dry to moist regions. Such a process was already proposed by Gray and Jacobson (1977) to explain the observed reinforcement of large convective systems at the end of the night. This circulation transports near-surface high moist static energy (MSE) from dry to moist regions. This MSE upgradient transport maintains high MSE in the moist region, helping to maintain deep convection there. In fact, in the CRM used in this study [System for Atmospheric Modeling (SAM); Khairoutdinov and Randall 2003], there is no self-aggregation without interactive radiation [unless the evaporation of rain is artificially suppressed (Muller and Bony 2015), a particular case which will not be discussed here]. Because of the idealized settings in which self-aggregation was discovered, its relevance to the real world is still debated. Notably, the aforementioned CRM studies used spatially and temporally constant and uniform SSTs.

The impact of SST anomalies on deep convection has already been widely studied in the literature (Tompkins 2001b; Kuang 2012; Ramsay and Sobel 2011; Sobel and Bretherton 2000). Tompkins (2001b) found in particular that a sudden inverting of an imposed SST anomaly leads to migration of the convective clusters over the warm anomaly. The migration of aggregated convective clusters over warm anomalies has been confirmed by other studies that used a slab ocean in order to have interactive SSTs (Coppin and Bony 2015; Grabowski 2006). Using a singlecolumn model (SCM) and CRM, Ramsay and Sobel (2011) and Wang and Sobel (2011) showed that precipitation rate increases over local warm SSTs and is determined by the temperature anomaly rather than by the mean SST. Daleu et al. (2017) confirmed this result using two adjacent SCMs with different SST. The SST difference, if large enough, can suppress convection in the cold column and strengthen it in the warm column. Notably, SST gradients can generate a large-scale circulation that can lead to a migration of deep convection toward the warmest SST.

Another type of surface temperature anomalies are tropical islands with different surface properties, which act as a surface forcing and change the intensity of convection (Crook 2001; Beringer and Tapper 2002) and thermal structure of the atmosphere (Cronin et al. 2015). Rainfall over tropical islands is larger than over the surrounding ocean (Cronin et al. 2015; Sobel et al. 2011; Qian 2008; Wang and Sobel 2017); however, the strength of the thunderstorms and precipitation depends on several factors such as the size of the islands, wind speed and direction, and the island's topography (Wang and Sobel 2017;
Crook 2001). Convective events over tropical islands show large diurnal variations; however, they build up an average ascent (Cronin et al. 2015).

Ocean mesoscale eddies (Chelton 2011) can also be associated with SST anomalies reaching a few degrees in cold-core cyclonic eddies or warm-core anticyclonic eddies. These persistent ocean eddies have typical radius varying with latitude, from a hundred to a few hundreds of kilometers in the tropics ( $\pm 20^{\circ}$ latitude), to around $50 \mathrm{~km}$ or less in midlatitudes. As a surface forcing, eddies can impact the atmosphere locally (Sugimoto et al. 2017) by enhancing low-level convergence and thus convective precipitation. Potentially, the eddies changes the cloudiness and wind field which can impact the large-scale circulation.

Whether and how such persistent SST anomalies, as an external forcing, can favor or suppress the aggregation of convection is, to our knowledge, still not well covered in the literature. In this paper, we investigate the aggregation response to an idealized, circular SST anomaly referred to as a "hot spot." We must emphasize that the aggregation forced by a hot spot, when it is the case, is not anymore "self-aggregation" but rather a forced aggregation. Of particular interest are the following questions:

- How does the presence of an ocean hot spot modify or enforce the aggregation process of the deep convection? And how does this modification depend on the hot spot radius and temperature anomaly?

- How does the hot spot impact the large-scale circulation?

- In the presence of a hot spot, how does the aggregation physics differ from the self-aggregation ones; specifically, does aggregation disappear in the absence of radiative feedbacks (known to be crucial for self-aggregation over homogeneous SST)?

The next section, section 2, describes the cloud-resolving model used and the experimental setup, as well as the metrics used to measure (self-)aggregation. Section 3 investigates the impact of the hot spot on convective aggregation, and the sensitivity to hot spot properties. Section 4 investigates whether radiative feedbacks are still necessary for aggregation to occur when a hot spot is present. Additionally, we derive a simple, two-box model to help comparison between the onset of self-aggregation and aggregation. In section 5 we briefly discuss the equilibrium phase, once aggregation has occurred. Conclusions are given in section 6.

\section{Model description and simulation design}

\section{a. Cloud-resolving model}

The CRM used is the model SAM version 6.11.1 (Khairoutdinov and Randall 2003). This model solves the anelastic equations of conservation of momentum, water (with six species present in the model, water vapor, cloud liquid, cloud ice, precipitating rain, precipitating snow, and precipitating graupel), and energy. The relevant energy for moist convection is the moist static energy, as it is conserved (approximately, i.e., neglecting viscous and subgrid-scale effects) under adiabatic processes including the phase change of water. More precisely in this model, the so-called frozen MSE is conserved during moist adiabatic processes, including the freezing of precipitation. The frozen MSE is given by 


$$
\mathrm{MSE}=c_{p} T+g z+L_{v} q_{v}-L_{f} q_{\mathrm{ice}},
$$

with the specific heat capacity of air at constant pressure $c_{p}$, temperature $T$, gravity $g$, height $z$, latent heat of evaporation $L_{v}$, water vapor mixing ratio $q_{v}$, latent heat of fusion $L_{f}$, and mixing ratio of all ice phase condensates $q_{\text {ice }}$.

The subgrid-scale turbulence is modeled using a Smagorinskytype parameterization, and we use the 1-moment microphysics formulation, following Bretherton et al. (2005) and Muller and Held (2012). Bulk formulas are used to compute surface fluxes. Further information about the model can be found in Khairoutdinov and Randall (2003).

Most simulations use interactive radiation, using the radiation code from the National Center for Atmospheric Research (NCAR) Community Atmosphere Model version 3 (CAM3; Collins et al. 2006). For simplicity, we neglect the diurnal cycle and use the daily mean incoming solar insolation of $413 \mathrm{~W} \mathrm{~m}^{-2}$ [same setting as Tompkins and Craig (1998)]. Studies of selfaggregation over the ocean with a diurnal cycle show that, quantitatively, a diurnal cycle can change the strength of the hydrological cycle, increasing the daily precipitation range. But qualitatively, beyond this daily modulation of amplitude, it does not seem to affect the fact that deep convection selfaggregates or not.

In some simulations, radiative feedbacks are turned off by homogenizing radiative cooling rates horizontally, at each height and time step, following Muller and Held (2012). Note that in that case, the domain average radiative cooling rates can still evolve in time.

\section{b. Experimental setup}

The model domain is square, doubly periodic in both horizontal directions $x$ and $y$. We run simulations with two domain sizes, $(288 \mathrm{~km})^{2}$ and $(576 \mathrm{~km})^{2}$ [except for one simulation shown in Fig. 1 with a smaller $(96 \mathrm{~km})^{2}$ domain]. The horizontal resolution is $3 \mathrm{~km}$ and the vertical grid spacing increases gradually with height, with the first level at $25 \mathrm{~m}$ and a resolution of $50 \mathrm{~m}$ close to the sea surface, reaching a vertical resolution of $500 \mathrm{~m}$ in the mid troposphere. There are 64 vertical levels which span $27 \mathrm{~km}$ in the vertical. This includes a sponge layer in the upper third of the domain (from $z=18$ to $27 \mathrm{~km}$ ) where the wind is relaxed to zero in order to reduce gravity wave reflection and buildup. No large-scale forcing or wind is imposed. We neglect Earth's rotation, a reasonable approximation in the tropics where the Coriolis parameter is small.

The initial conditions for the different mean SSTs (horizontal mean SSTs in our simulations with and without hotspot) are obtained from a smaller domain run with the corresponding SST at RCE $\left[(96 \mathrm{~km})^{2}\right.$ run to 50 days], then using time and domain averaged profiles of the last 5 days. We run two different types of simulations: simulations with a uniform and constant sea surface temperature that we refer to as ocean experiments, and simulations with a warm temperature anomaly referred to as hot spot experiments. The hot spot is a circular area with a higher temperature than the surrounding ocean, located at the center of the domain. A given hot spot simulation will be defined by its temperature anomaly $d T$ and its radius $R$ so that, for example, simulation dT5R60 is for a hot spot with a
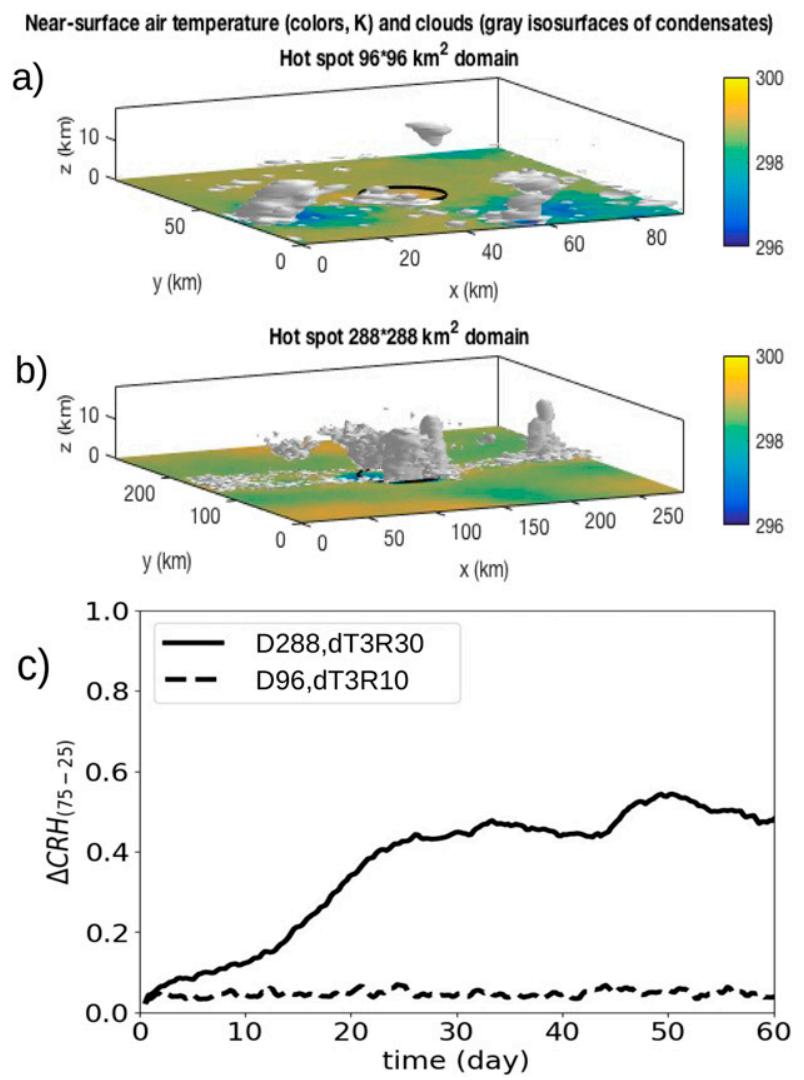

FIG. 1. Snapshots of near-surface air temperature (colors, K) and cloud water (gray shades) from two simulations with a hot spot in the center of the domain (circle) for (a) a domain size $96 \times 96 \mathrm{~km}^{2}$ and (b) $288 \times 288 \mathrm{~km}^{2}$. (c) Time evolution of the aggregation index for those two simulations.

temperature anomaly of $5 \mathrm{~K}$ and a radius of $60 \mathrm{~km}$. The top two panels of Fig. 1 show snapshots of near-surface air temperature and cloud water for two simulations with a different domain size and hot spot radius. This illustration shows that, although there is some organization of convection on the small domain in the presence of a hot spot, the self-aggregation of convection surrounded by extremely dry air only occurs in the largedomain simulation. This is well captured by the metrics used to quantify the degree of aggregation described next and shown in Fig. 1c. In the following, in both ocean and hot spot experiments, we also investigate the role of radiative feedbacks by repeating some simulations with homogenized radiation.

\section{c. Aggregation metrics}

The convective aggregation is associated with progressive drying of the dry environment surrounding deep clouds, and progressive moistening of the moist region where deep convection occurs. This leads to increased horizontal moisture variability. Thus a common index for self-aggregation is the difference between the 75th and 25th percentiles of precipitable water, $\Delta \mathrm{PW}_{75-25}$ (Muller and Held 2012; Muller and Bony 2015). Since here we will compare simulations with different SSTs, we will use precipitable water normalized by the 
a

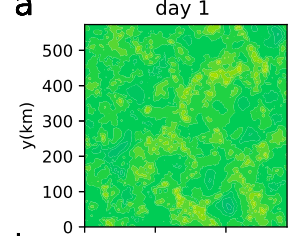

b

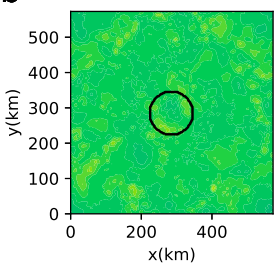

day 11
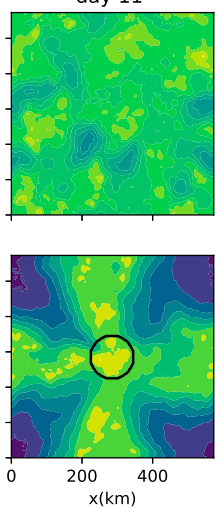

day 21
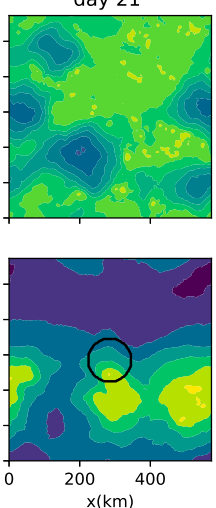

day 31
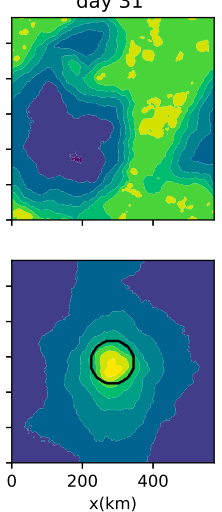

day 41
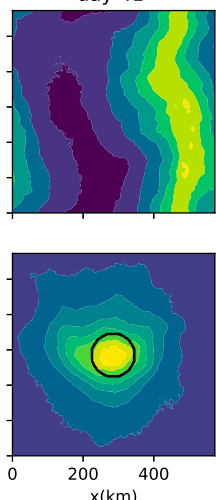

day 51

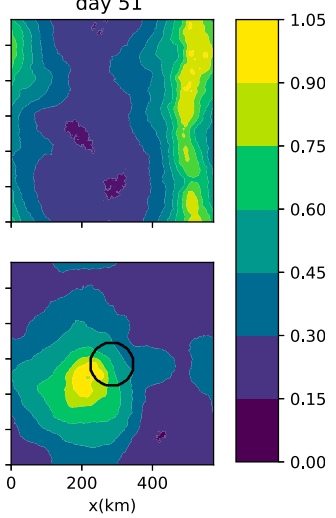

FIG. 2. Snapshots of CRH for simulations with (a) a uniform surface temperature and (b) a hot spot with a SST anomaly of $5 \mathrm{~K}$ and a radius of $60 \mathrm{~km}$. The black circle shows the hot spot boundary. For both simulations the domain average SST is $300 \mathrm{~K}$ and the domain size is $576 \times 576 \mathrm{~km}^{2}$.

saturation water vapor path, i.e., we will use column relative humidity, CRH (Wing and Cronin 2016),

$$
\mathrm{CRH}=\frac{\int q_{v} \rho d z}{\int q_{v, \mathrm{sat}} \rho d z},
$$

where $q_{v \text {,sat }}$ denotes the saturation water vapor mixing ratio, $\rho$ density, and the vertical integration done over the troposphere. Our aggregation index is the difference between the 75th and 25th percentiles of column relative humidity, $\Delta \mathrm{CRH}_{75-25}$. Figure 1 illustrates the increase of this index (bottom panel) in the simulation that aggregates (middle panel).

In SAM, self-aggregation has been shown to start with the strengthening and the expansion of a dry patch, becoming drier and larger. This dry region, devoid of deep convection, was sometimes referred to as the "radiative dry pool" (Coppin and Bony 2015; Zuidema et al. 2017), as it is believed to be radiatively driven. The dry patches are thus of primary importance, as the self-aggregation of convection can eventually result from the confinement of the deep convection in a restricted region because of the expansion of a dry patch in our doubly periodic geometry. In the following, the dry patch is defined as the area where the CRH is below the 25 th percentile.

\section{Hot spot impact on aggregation of deep convection}

Here, we first investigate how the presence of a hot spot impacts the aggregation of convection in the presence of radiative feedbacks. Of particular interest is whether the aggregation is faster, and whether the deep convection area ends up being localized over the hot spot.

\section{a. Results without and with hot spot at different SSTs}

The top row of Fig. 2 shows the CRH maps in a control ocean experiment with a mean SST of $300 \mathrm{~K}$ at different times started from homogeneous conditions. We observe the typical evolution of self-aggregation: the appearance of a dry patches after a few days (day 11) and thus the extension and merge of these dry patches into a single patch (day 31 ). At day 41, the $\mathrm{CRH}$ in the dry region reaches extremely low values, and convection and moisture are confined to a small part of the domain. After day 41 , the moist patch shrinks to a narrow region surrounded by a very dry environment. The increased spatial moisture variability between dry and moist regions, largely due to enhanced drying, is also visible in $\Delta \mathrm{CRH}_{75-25}$ (Fig. 3a). It increases up to day 40 and then starts to decrease slowly. With further progress of aggregation, the high $\mathrm{CRH}$ region shrinks to a circular area smaller than $25 \%$ of the domain, thus $\mathrm{CRH}_{75}$ decreases, leading to the decrease of the aggregation index.

Self-aggregation over fixed SSTs is known to depend on the domain mean SST. Using the same SAM model, Wing and Emanuel (2014) find that warm SSTs favor aggregation, while Coppin and Bony (2015) find in a GCM that self-aggregation is surprisingly favored both for SSTs larger than $295 \mathrm{~K}$ or smaller than $285 \mathrm{~K}$. In very cold snowball simulations, aggregation can also occur (Abbot 2014), though in that case a weak wind shear can prevent the aggregation. The exact relation between an average SST and the self-aggregation response is hence still unclear, but the general consensus is that self-aggregation is favored at warm SSTs (Emanuel et al. 2014). Consistently, we find that for a colder SST of $290 \mathrm{~K}$ aggregation does not occur, and that the aggregation speed increases regularly with the SST for SST values between 295 and $305 \mathrm{~K}$ (Fig. 3a).

Simulations with the same mean SST, but with different hot spot characteristics are performed to analyze the role of the SST anomaly on the convective aggregation. Here the domainmean SST is kept constant at $300 \mathrm{~K}$ in order to isolate the effect of the hot spot temperature anomaly. Consequently, the surrounding ocean temperature is slightly lower than $300 \mathrm{~K}$ in the hot spot simulations. However, it has been argued in previous studies (Ramsay and Sobel 2011; Wang and Sobel 2011) that the control parameter is the SST anomaly $(d T)$ and not the absolute SST, at least for a reasonable temperature change. Figure $2 \mathrm{~b}$ shows the hot spot experiment dT5R60 $(d T=5 \mathrm{~K}$ and $R=60 \mathrm{~km}$ ). Spatially, the main aspects of aggregation in the presence of a hot spot are similar to the ocean experiment, with a progressive expansion of dry regions. The aggregation is however much faster with the hot spot and the convection is 

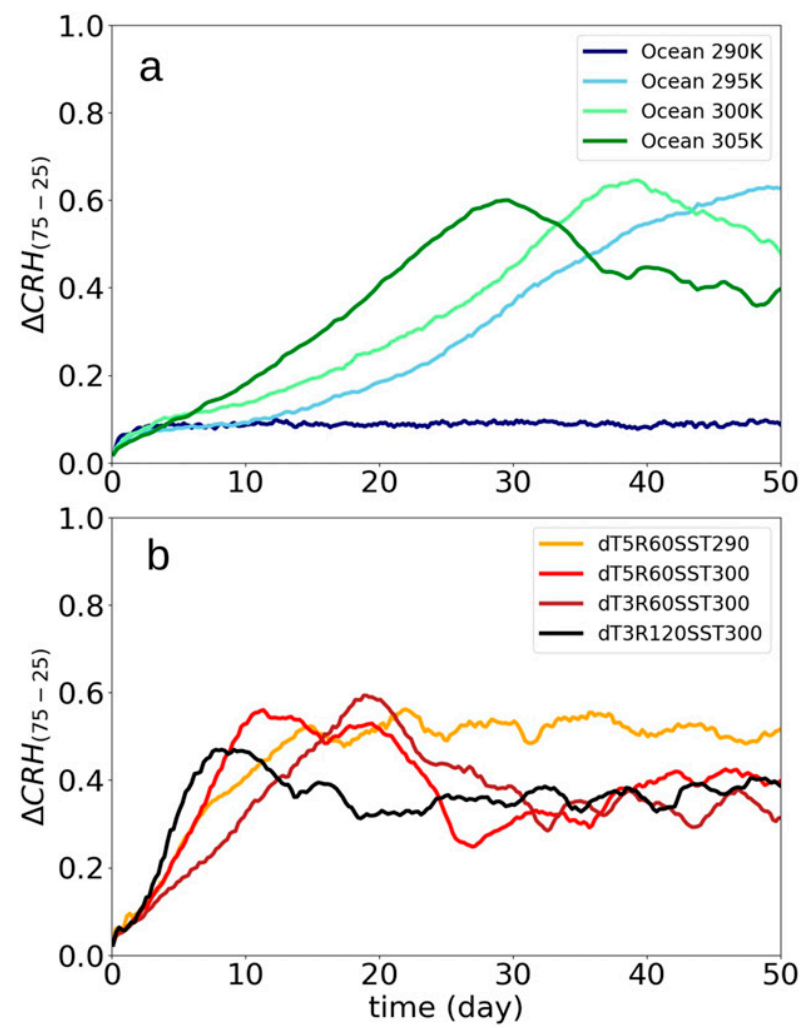

FIG. 3. Time evolution of the aggregation index for simulations with full radiative feedback for (a) simulations with a uniform surface temperature (referred to as "Ocean"; see section $2 b$ for a detailed description of the simulations) and (b) simulations with a hot spot of different sizes and SST anomalies.

eventually organized over or near the hot spot. Note that the location of the aggregation is not stable, and whether the aggregated convective cluster stays over the hot spot depends on hot spot radius and temperature. If the hot spot is sufficiently large and/or warm, it sustains the convective cluster over it, otherwise, it does not necessarily stay over the hot spot after its formation. We will discuss this in more detail in section 5 .

Looking at the aggregation index (Fig. 3b), the maximum aggregation is in fact reached after only 10 days in dT5R60 compared to 40 days in the ocean simulation at $300 \mathrm{~K}$. Thus, the presence of a hot spot may accelerate the aggregation by a factor of 4. However, the aggregation is much faster with a hot spot. When the aggregation is fully reached, the aggregation index is fairly comparable between the simulations with and without a hot spot. The hot spot temperature anomaly plays a significant role in accelerating or enforcing the aggregation, as can be seen in Fig. 3b. For dT5R60 the aggregation index reaches a maximum after only 10 days while for dT3R60 the maximum is reached in 20 days. Thus, the aggregation speed is favored by larger hot spot temperature anomaly. The hot spot size also plays a role with a maximum aggregation index reached in less that 10 days for dT3R120. Therefore, the larger the hot spot, the faster the aggregation. Note though that for very large hot spots relative to the domain size (see below), this cannot hold anymore. A hot spot can also extend the range of SSTs for which an aggregation occurs. For example, with an average SST of $290 \mathrm{~K}$, there is no self-aggregation for uniform SST (Fig. 3a), but the dT5R60 experiment at $290 \mathrm{~K}$ aggregates even faster than uniform ocean simulations at $305 \mathrm{~K}$ (Fig. 3b).

\section{b. Development of a large-scale circulation}

Here, we hypothesize that the presence of the hot spot favors and accelerates the formation of a large-scale circulation that triggers the onset of convective aggregation, and thus extends the range of SSTs at which aggregation occurs.

To explain the acceleration of aggregation with a hot spot, we look at virtual potential temperature $\left(\theta_{v}\right)$ anomaly. In the free troposphere, gravity waves remove horizontal $\theta_{v}$ anomalies very efficiently (Bretherton and Smolarkiewicz 1989; Ruppert and Hohenegger 2018) so that $\theta_{v}$ profile above the boundary layer is fairly uniform over the domain especially when it is averaged over a few hours. So the main source of instability is the buoyancy anomaly in the boundary layer. Figure 4 shows $\theta_{v}$ anomaly averaged over the boundary layer for the ocean experiment at SST $=300 \mathrm{~K}$ at day 31 and the hot spot experiment dT5R60 with mean SST equal to $300 \mathrm{~K}$ at day 11 (Fig. 2 shows the CRH evolution for these two simulations). We compare these two days as the aggregation index and the fraction of area covered with low (high) $\mathrm{CRH}$ are comparable between the two simulations. In general there is a positive $\theta_{v}$ anomaly in moist areas (except directly below clouds where cold pools result from the partial evaporation of rain), that enforces convergence of low-level air toward the moist area. Consistent with the faster aggregation, the $\theta_{v}$ anomaly is larger over the hot spot. The value of $\theta_{v}$ depends on both temperature and water vapor. In both the ocean and hot spot simulations, the moisture contribution to the $\theta_{v}$ anomaly in moist regions is positive. But the temperature contribution is smaller in the ocean experiment. In the hot spot simulations, over the hot spot, both temperature and moisture have a positive contribution to $\theta_{v}$ resulting in a slightly larger $\theta_{v}$ anomaly and a stronger instability over the hot spot that leads to stronger convection.

The corresponding pressure gradient at the first few levels enforces a convergence of moisture toward the moist region. With a hot spot, the pressure gradient is larger and it stays over the hot spot. This convergence favors convection over the hot spot by transporting low-level moist air and by providing energy to lift the air above the hot spot. Additionally, the convergence of moisture removes moisture from the environment and inhibits convection there. This process (low-level transport of moisture toward the moist region) thus seems common to both self-aggregation and aggregation but is stronger in the latter case. There is a difference though: in aggregation with a hot spot, it is the strength of the upward mass flux over the hot spot which seems to control the large-scale circulation and thus the aggregation speed. Ascent over the hot spot forces compensating subsidence in the environment, which dries the troposphere and results in further suppression of convection there and enhancement of moisture transport toward the hot spot. This upward motion over the hot spot and thus subsidence in the environment, is partly a consequence of our periodic 


\section{a}

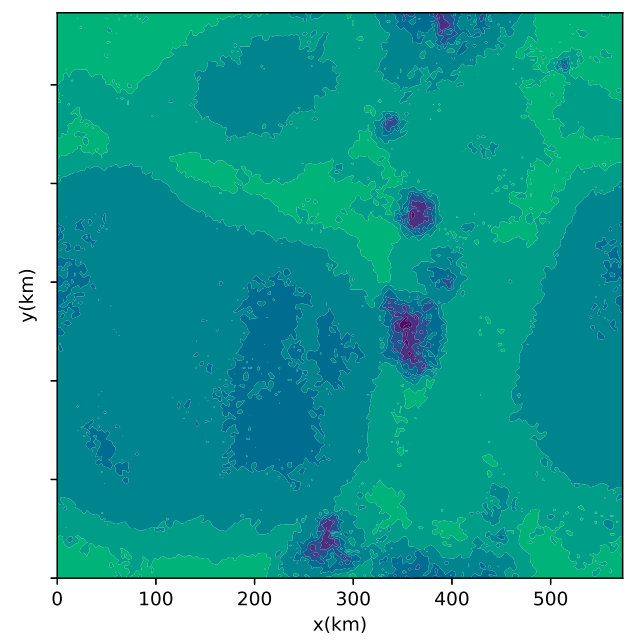

b

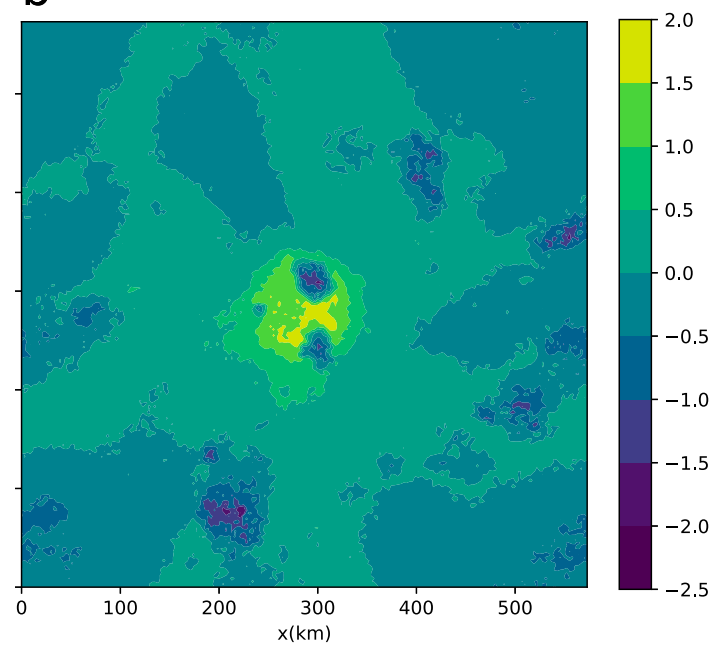

FIG. 4. $\theta_{v}$ anomaly averaged over the boundary layer (from the surface to $1000 \mathrm{~m}$ ) for (a) day 31 of ocean experiment at $300 \mathrm{~K}$ and (b) day 11 of hot spot experiment dT5R60 and mean SST $=300 \mathrm{~K}$.

boundary conditions, and it builds up a large-scale circulation that accelerates the aggregation. Instead, with selfaggregation, it has been hypothesized that it is the subsidence in dry regions which initiates and controls the large-scale circulation, and thus the self-aggregation speed. This development of a large-scale circulation will be further investigated in the next section.

A natural question then, is whether the large-scale circulation enforced by the hot spot can be maintained even in the absence of the hot spot, solely by internal self-aggregation feedbacks. The sensitivity of self-aggregation to initial conditions is well documented. Aggregated states that are imposed as initial conditions can persist, even under conditions which do not favor the spontaneous self-aggregation from homogeneous initial conditions (Khairoutdinov and Emanuel 2010; Muller and Held 2012). To investigate whether the hot spot aggregation exhibits hysteresis, we repeat the dT5R60 with SST = $290 \mathrm{~K}$ simulation, which does not self-aggregate without hot spot, for 30 days, and then remove the hot spot (by simply setting $d T$ to zero) and run for another 30 days. The aggregated cluster spreads over the domain and disaggregates. Therefore the aggregation is not maintained without the hot spot in this case.

\section{Convective aggregation without radiative feedbacks}

\section{a. Hot spots with or without radiative feedbacks}

Radiative feedbacks have been shown by many studies to be necessary for convective self-aggregation, at least for typical tropical SSTs around $300 \mathrm{~K}$ (Wing et al. 2017). The balance between radiative cooling and subsidence warming in dry regions (Mapes 2001) creates a positive feedback that results in radiatively enhanced subsidence and drying of already dry regions. Sensitivity studies show that removing radiative feedbacks, by homogenizing radiative cooling rates, prevents the self-aggregation. Here we test the occurrence of aggregation without radiative feedbacks in hot spot experiments, listed in Table 1.

Comparing the dT5R60 simulation with (Fig. 2b) or without (Fig. 5a) radiative feedbacks, we see that homogenizing the radiation prevents aggregation for a hot spot radius of $60 \mathrm{~km}$. However, increasing the hot spot radius to $70 \mathrm{~km}$ (Fig. 5b) yields aggregation even without radiative feedback. For $R=$ $70 \mathrm{~km}$, the aggregation is very slow, but it becomes much faster at larger radii (Fig. 6). It is worth noting that simulations with $R=70$ and $80 \mathrm{~km}$ give a banded aggregation. For larger hot spots, a circular aggregation of the convection develops in a few days, with a maximum aggregation index reached in less than 10 days with $R=180 \mathrm{~km}$. This is fast compared to typical overturning time scale of the atmosphere (Grabowski and Moncrieff 2001), suggesting that the circulation between dry and moist regions is greatly accelerated by the presence of the SST anomaly. By reducing this anomaly to $3 \mathrm{~K}$ instead of $5 \mathrm{~K}$, there is no convective aggregation, even for a radius of $80 \mathrm{~km}$ (Fig. 6). A persistent SST anomaly can thus clearly trigger a convective aggregation in SAM, even without radiative feedbacks.

TABLE 1. List of all the simulations with homogenized radiation. Shown are the hot spot radius, the fractional area covered by it (with one digit for values below $10 \%$ ), its temperature anomaly $(d T)$, ocean temperature, and domain mean SST.

\begin{tabular}{ccccc}
\hline \hline $\begin{array}{c}\text { Hot spot } \\
\text { radius }(\mathrm{km})\end{array}$ & $A^{\mathrm{hs}} /\left(A^{\mathrm{env}}+A^{\mathrm{hs}}\right)(\%)$ & $d T(\mathrm{~K})$ & $\mathrm{SST}^{\mathrm{env}}(\mathrm{K})$ & $\overline{\mathrm{SST}}(\mathrm{K})$ \\
\hline 60 & 3.4 & 5 & 299.83 & 300 \\
65 & 4.0 & 5 & 299.80 & 300 \\
70 & 4.6 & 5 & 299.77 & 300 \\
80 & 6.1 & 5 & 299.69 & 300 \\
80 & 6.1 & 3 & 299.81 & 300 \\
180 & 31 & 5 & 298.46 & 300 \\
220 & 46 & 5 & 297.70 & 300 \\
285 & 77 & 5 & 296.15 & 300 \\
\hline
\end{tabular}


a

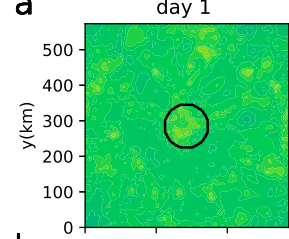

b

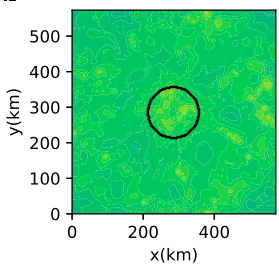

day 11
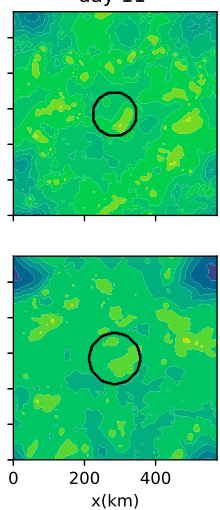

day 21
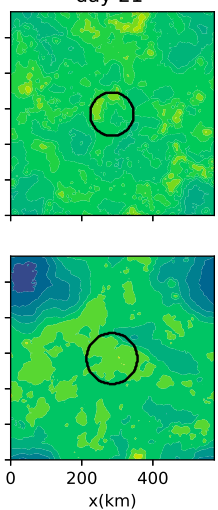

day 31
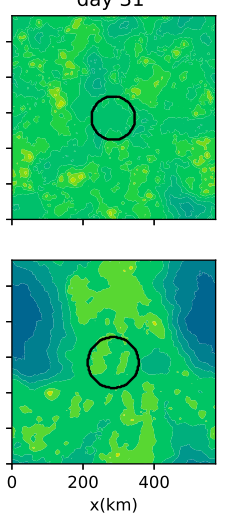

day 41
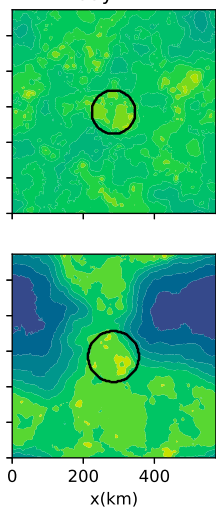
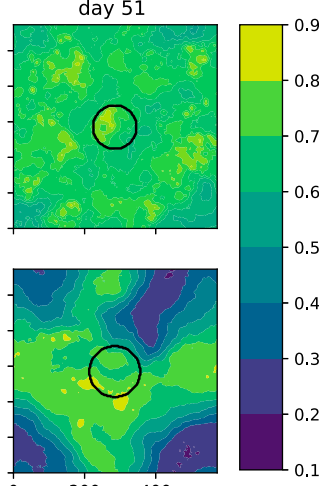

$\mathrm{x}(\mathrm{km})$

FIG. 5. Snapshots of CRH for hot spot simulations with homogenized radiation for (a) SST anomaly of $5 \mathrm{~K}$ and a radius of $60 \mathrm{~km}$ and (b) SST anomaly of $5 \mathrm{~K}$ and a radius of $70 \mathrm{~km}$. The black circle shows the hot spot boundary. For both simulations, the domain average SST is $300 \mathrm{~K}$ and the domain size is $576 \times 576 \mathrm{~km}^{2}$.

This aggregation requires a minimum size and amplitude of the SST anomaly, and is faster for warm and large hot spots. To clarify the physical processes responsible for convective aggregation in that case, we look in the next section at the largescale circulation in more detail, in particular the subsidence in the dry regions.

Note that because we keep the mean SST constant, changing the hot spot radius $R$ and temperature anomaly $d T$, also changes the temperature outside the hot spot and the absolute temperature of the hot spot (both reduced to keep the domain mean SST constant). To verify that the leading-order parameter determining the onset and speed of aggregation is the hot spot temperature anomaly $d T$, not its absolute temperature, we redo some of the simulations keeping the temperature equal to $300 \mathrm{~K}$ outside the hot spot, and simply adding a hot spot with $d T=5 \mathrm{~K}$ to the domain (so that the domain mean SST is now larger than $300 \mathrm{~K}$ ). We find that the speed of aggregation, based on the aggregation index, is similar, and is determined to leading order by $d T$. This gives us confidence that the hot spot temperature anomaly is indeed the main control parameter, not its absolute temperature.

Previous studies showed that the self-aggregation of convective clouds is sensitive to initial conditions so that just by changing initial noise, which is small compared to the initial condition, the aggregation onset may delay or hasten. To check the robustness of our results regarding the timing of the onset and the speed of aggregation, we ran two small ensembles of five members for dT5R70 and dT5R80 with homogenized radiation, using different initial noise. The ensemble simulations show that the aggregation onset and speed do not vary significantly among the members, in particular the $R=80 \mathrm{~km} \mathrm{sim}$ ulations are all faster than the $R=70 \mathrm{~km}$. This suggests that the aggregation speed is set mostly by the hot spot forcing, and dependency on the initial conditions is small.

\section{b. Two-box model: Pulled or pushed aggregation?}

Here we further investigate the mechanisms involved in the aggregation of the convection in the absence of radiative feedbacks (Fig. 6). Given the potential importance of expansion and strengthening of the dry patch for the onset of convective aggregation (consistent with the drying in Fig. 6b), we will interpret the results in light of a conceptual, two-box model with a dry and a moist region, illustrated in Fig. 7. In the moist
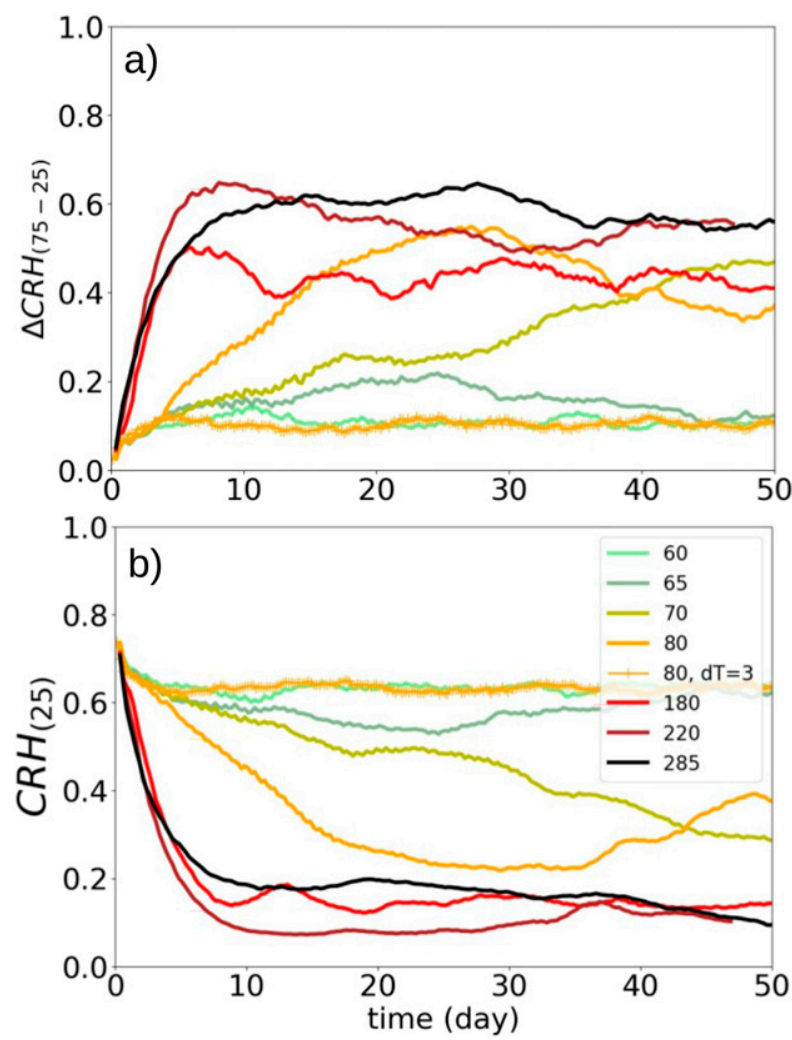

FIG. 6. Time evolution of (a) the aggregation index and (b) CRH averaged over driest quartile for different hot spot radii for simulations with homogenized radiation. All the simulations have a domain size of $576 \times 576 \mathrm{~km}^{2}$ and a hot spot SST anomaly of $5 \mathrm{~K}$ except for one simulation with a radius of $80 \mathrm{~km}$ and an SST anomaly of $3 \mathrm{~K}$. 

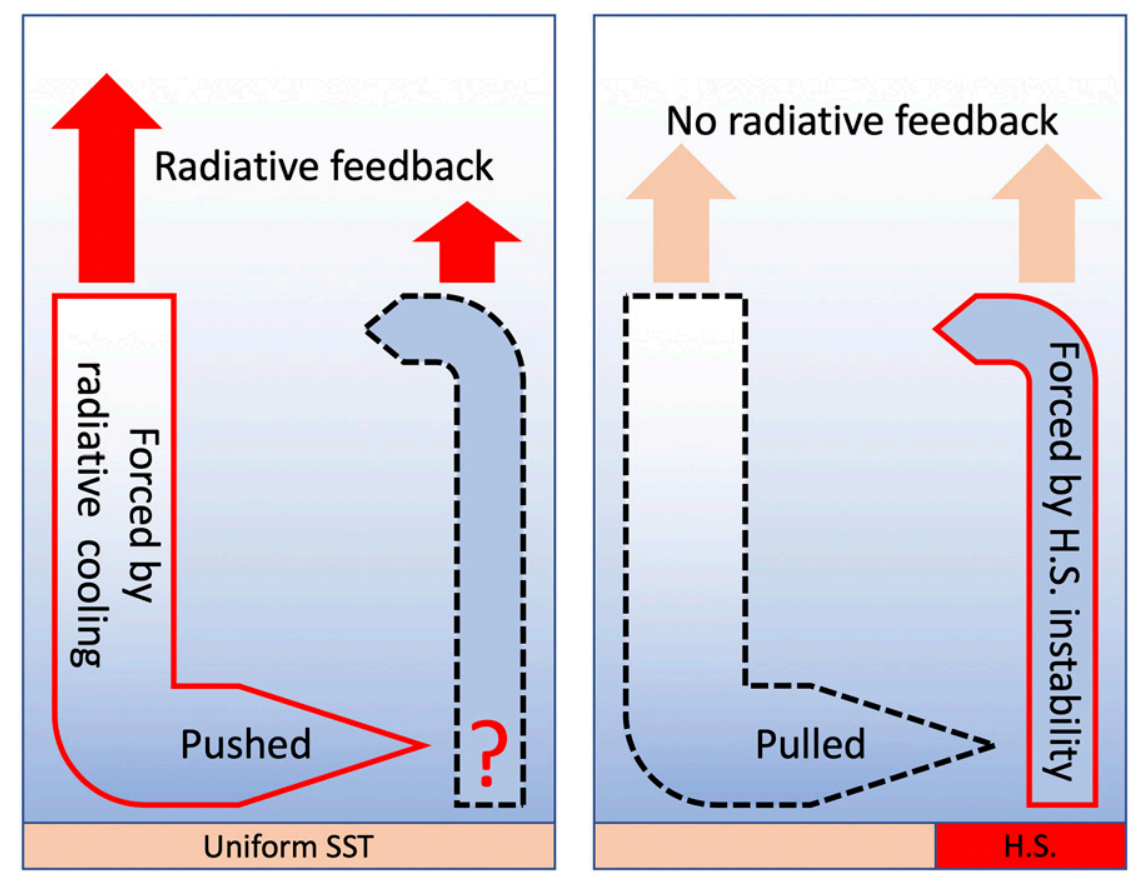

FIG. 7. Schematic two-box model representing either a self-aggregation by radiative feedbacks or an aggregation forced by a hot-spot-induced circulation. (a) Self-aggregation by radiative feedbacks is caused by a progressive expansion of a dry subsidence region under the effect of a strong radiative cooling, "pushing" the low-level moisture toward a constricted moist and warm convective region. (b) The aggregation is due to the large-scale circulation induced by the hot spot persistent SST anomaly, "pulling" the moisture toward the warm anomaly.

region, there is upward motion in deep convection. In the dry region, there is subsidence and no deep convection (thus no latent heat release). Therefore, given the small horizontal gradients of temperature in the tropics [so-called weak temperature gradient approximation (WTG); Sobel et al. 2001], to first order the temperature equation for a given pressure level (500 $\mathrm{hPa}$ in the following) yields

$$
\begin{aligned}
\frac{\partial T}{\partial t}+\Gamma w^{\mathrm{dry}} & =Q_{\mathrm{rad}} \\
\Rightarrow w^{\mathrm{dry}} & =\frac{Q_{\mathrm{rad}}-\partial T / \partial t}{\Gamma},
\end{aligned}
$$

where $w^{\mathrm{dry}}$ is the (negative) subsidence velocity $\left(\mathrm{m} \mathrm{s}^{-1}\right), Q_{\mathrm{rad}}$ the (negative) radiative cooling $\left(\mathrm{K} \mathrm{s}^{-1}\right)$, and

$$
\Gamma=\frac{T}{\theta} \frac{d \theta}{d z}
$$

the static stability ( $\theta$ denotes potential temperature in $\mathrm{K})$. At equilibrium (i.e., $\partial T / \partial t=0$ ), there is a balance between subsidence warming and radiative cooling in the dry environment. We nevertheless retain the temperature term $\partial T / \partial t$ in anticipation of $w^{\text {dry }}$ that it may be important during the onset of selfaggregation, before equilibrium is reached. Recall that in these simulations without radiative feedback, the radiative cooling rates are homogenized in space, but is allowed to evolve in time.
As stated in the introduction for self-aggregation with radiative feedbacks, the stronger radiative cooling in dry regions compared to the moist regions causes further subsidence drying and generates a circulation that "pushes" the moisture toward the deep convection area (Fig. 7). Thus selfaggregation is rather a self-confinement of moisture, as dry regions expand and strengthen, pushing the convection in a small part of the domain in our doubly periodic geometry. In the hot spot aggregation, however, the hot spot increases the convective instability and leads to deep convection localized over the hot spot. Warmer and moister low-level conditions over the hot spot increases the convective instability compare to the environment if we assume that the free troposphere temperature is horizontally homogeneous (Bretherton and Smolarkiewicz 1989). This generates a large-scale circulation with upward motion over the hot spot and subsidence in its environment, yielding subsidence drying and convectively suppressed conditions in the region surrounding the hot spot. The moisture is thus "pulled" in the convective region by the large-scale circulation induced by the convective instability over the hot spot.

The aggregation may be separated into two different phases (Fig. 6): the aggregation onset phase where dry regions expand and dry further, and the equilibrium phase when aggregation is well established and the simulation is statistically in equilibrium. The mechanisms which govern aggregation at each of these phases might be different (Muller and Held 2012). For instance, Wing and Emanuel (2014) find that in the onset 

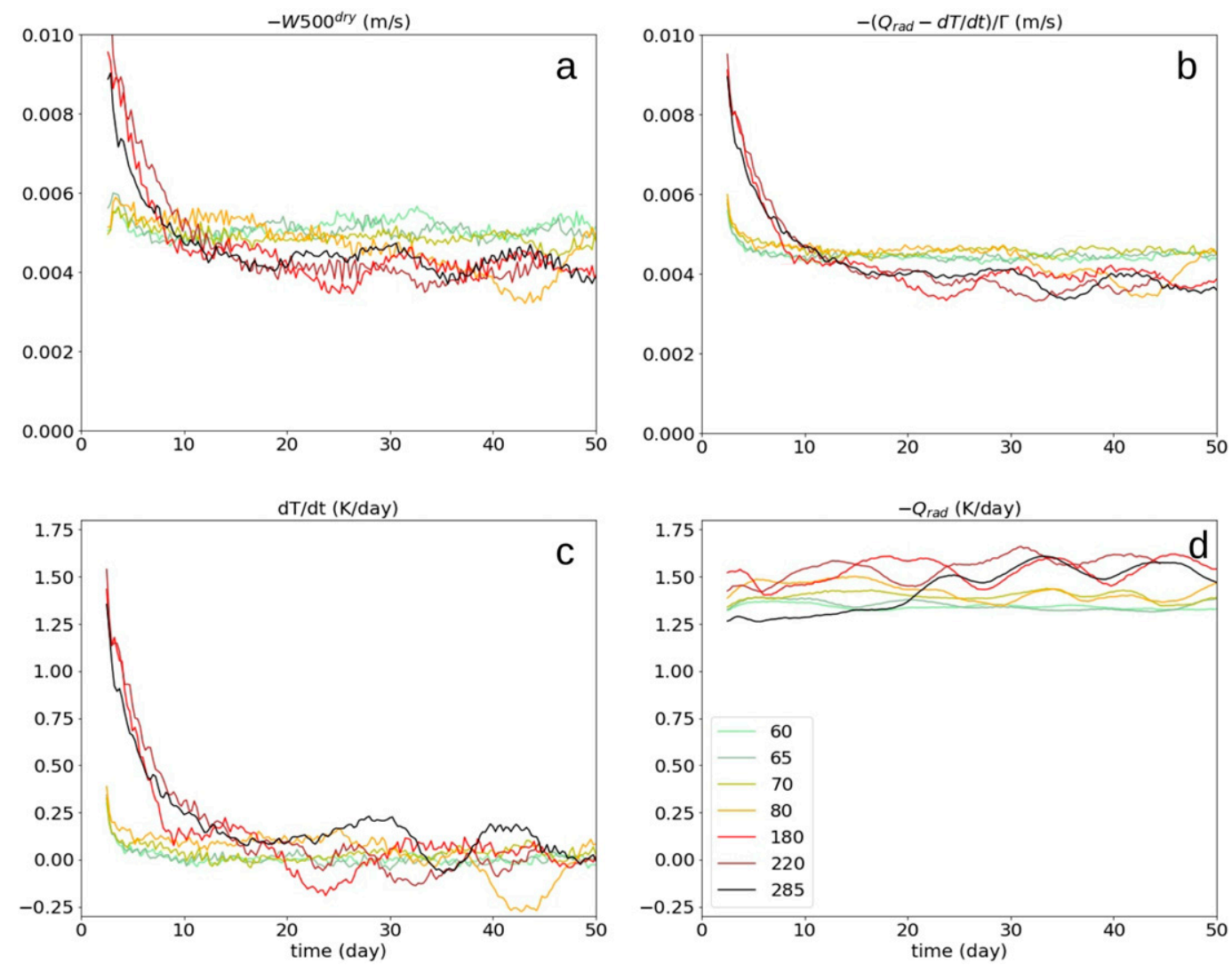

FIG. 8. Time evolution of atmospheric parameters at $500 \mathrm{hPa}$ averaged over the dry patch for different hot spot sizes: (a) vertical velocity, (b) the right-hand side of Eq. (4), (c) the time derivative of temperature, and (d) radiative cooling. The domain average SST is $300 \mathrm{~K}$ and the domain size is $576 \times 576 \mathrm{~km}^{2}$.

phase, surface latent heat fluxes act as a positive feedback largely due to enhanced latent heat fluxes in the moist region, while in the equilibrium phase the aggregation is opposed by enhanced surface fluxes in dry regions. Previous studies using the SAM model with homogeneous SST show that the radiative feedback is necessary for both the onset and the maintenance of aggregation, so that homogenizing the radiation profile even after the formation of aggregation leads to a nonaggregated convection. We showed above that a persistent SST anomaly can generate and sustain aggregation even with homogenized radiation. In the following sections we further focus on the hot spot simulations with homogenized radiation (Fig. 6). We analyze first the aggregation processes by considering separately dry and moist regions and by focusing on the aggregation onset phase. The equilibrium state will be addressed later in section 5 . We define the onset phase as the time between the beginning of the simulation and the first maximum of the aggregation index. The onset phase varies from less than 10 days to more than 50 days for the simulations considered in Fig. 6 and Table 1. Figure $6 \mathrm{~b}$ shows that the aggregation index is mostly driven by the $\mathrm{CRH}$ values in the dry patches $(\mathrm{CRH}<25$ th percentile).

\section{c. The aggregation onset phase}

The strength of the subsidence in the dry patch is characterized by its average vertical velocity at $500 \mathrm{hPa}\left({\overline{W_{500}}}^{\text {dry }}\right)$. Our hypothesis is that the subsidence strength is correlated with the aggregation onset and time scale. Stronger subsidence outside the hot spot leads to an enhanced subsidence drying in dry regions, this is an important process that is mostly driven by the positive radiative feedback in the self-aggregation, but it is driven here only by the enhanced vertical motion over the hot spot (Fig. 7). Consistent with this hypothesis, at the beginning of the simulations, the subsidence over the dry patch is larger for larger hot spots (Fig. 8a). This can be interpreted as a very fast response to the convective activity over the hot spot giving a strong subsidence over the surrounding cold ocean region. This response, much faster for larger hot spots, is largely due to the fact that the initial conditions of the atmosphere (based on a SST of $300 \mathrm{~K}$ ) enhanced the convective instability over the hot spot. This plays a role in the aggregation speed, in a manner that may be exaggerated in regard to a hot spot formation related, for example, to the diurnal surface temperature warming over an island. In that case, our results suggest that the adjustment is too slow (a few days) for such a diurnal variation to reach an equilibrium. Once the aggregation progresses, for hot spot radius larger than $70 \mathrm{~km}, \bar{W}_{500}$ dry becomes progressively weaker so that by the end of the aggregation onset phase, it becomes even weaker than for simulations without aggregation. 

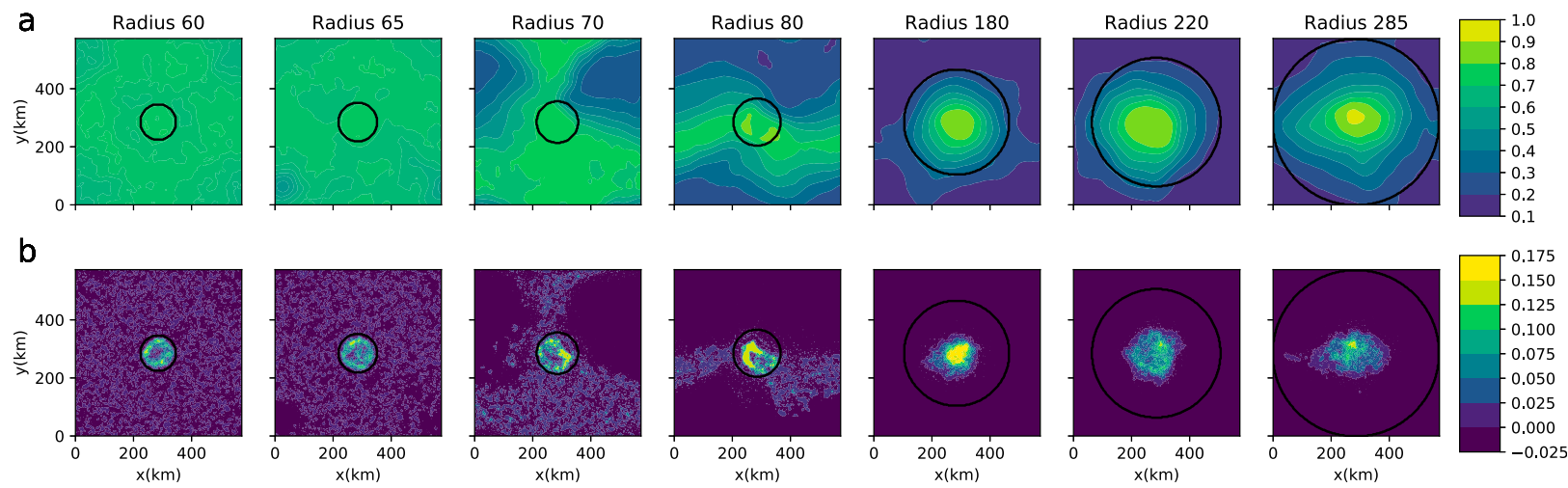

FIG. 9. (a) CRH and (b) W500 ( $\mathrm{m} \mathrm{s}^{-1}$ ) averaged between day 35 and day 50 of the simulation for hot spots of different sizes. The domain average SST is $300 \mathrm{~K}$, the hot spot SST anomaly is $5 \mathrm{~K}$, and the domain size is $576 \times 576 \mathrm{~km}^{2}$. The black circle shows the hot spot.

Equation (4) gives a good estimate of the evolution of the actual $\bar{W}_{500}$ dry (Fig. 8 b) that makes it possible to analyze further the contributions of the radiative cooling term $Q_{\mathrm{rad}}$ and of the warming term $\partial T / \partial t$ in the weakening of the subsidence over the dry patch (Figs. 8c,d). The difference in the time evolution of the subsidence is largely controlled by the warming term $\partial T / \partial t$ and not by $Q_{\text {rad }}$ during the aggregation onset phase. The warming term $\partial T / \partial t$ is large as the domain is adjusting to the warmer condition over the hot spot. The adjusting time is about 10 days for large hot spots. We note that this warming term is much smaller if we use atmospheric initial conditions corresponding to the hot spot temperature. The larger temperature above the hot spot yields warmer atmospheric temperatures there, which are progressively impressed on the whole domain through compensating subsidence and via propagating gravity waves (Bretherton and Smolarkiewicz 1989). As shown in Fig. 8, this effect is stronger for larger hot spots for which the term $\partial T / \partial t$ decreases dramatically during the aggregation onset phase. For large hot spots, $Q_{\text {rad }}$ is slightly larger at the end of the aggregation onset phase, showing the effect of a well organized dry patch compared to simulations without organized convection.

This moisture "pulling" leading to convective aggregation is associated to different overturning time scales in these simulations, with typically faster aggregation for larger hot spots. Note however that for $R=285 \mathrm{~km}$, the subsidence is found to be slightly smaller compared to $R=180 \mathrm{~km}$ or $R=220 \mathrm{~km}$, in good agreement with a longer aggregation onset phase (Fig. 6). For $R=285 \mathrm{~km}$, the subsidence is smaller because the potential upward mass flux over the hot spot is too large to be compensated by subsidence outside of the hot spot, so that a relatively large part of the hot spot is included in the subsiding region.

Thus, the aggregation is closely related to the large-scale circulation, as measured by the subsidence velocity in dry regions. The larger fractional area covered by the hot spot the larger $\bar{W}_{500}$ dry is. This can be well seen in Fig. 8 a.

The decrease of $\bar{W}_{500}$ dry during the aggregation onset phase for large hot spots is caused by the initial transient warming [Eq. (4) and Fig. 8c]. Eventually, $\bar{W}_{500}$ dry becomes nearly constant in time, as the equilibrium is reached. Then the main balance in dry regions is between subsidence warming and radiative cooling $(\partial T / \partial t \approx 0)$. The warming-induced enhanced static stability [large $\Gamma$ in Eq. (4)] reduces the subsidence velocity in aggregating simulations (Fig. 8a). Thus the vertical subsiding velocities in dry regions of aggregated simulations become smaller than nonaggregating ones once equilibrium is reached. This is how the expansion and strengthening of the dry patch is halted and equilibrium is reached, despite the stronger radiative cooling rates. This equilibrium phase will be further analyzed in the following section.

\section{Equilibrium phase}

Here we investigate how the strongest convective cells and updrafts are distributed in the equilibrium phase, and whether the aggregated cluster stays over the hot spot. To study the equilibrium state, we consider a period of 15 days starting at day 35 and ending at day 50 for which the simulations already reached the equilibrium phase (Fig. 6), except for a hot spot of $\mathrm{R} 70$ for which we look at the last 5 days as this period is closer to the equilibrium.

Figure 9 shows $\mathrm{CRH}$ and $W_{500}$ fields averaged over this period. For $R \leq 65 \mathrm{~km}$, there is no aggregation visible on the $\mathrm{CRH}$ field or detected by aggregation index; however, $W_{500}$ is much stronger over the hot spot compared to its environment. For $R=70$ the aggregation is still on progress. The CRH map of this simulation shows both dry and moist area; however, similar to $R=60$ and 65 , the convection over the hot spot is much stronger than over the environment. For $R=80 \mathrm{~km}$, the convection is not totally centered on the hot spot for this equilibrium phase. For the largest hot spots, the region of large $\mathrm{CRH}$ is well centered on the center of the hot spots. The concentration of the moist patch over the hot spot for aggregated simulations is not systematic. In the simulations with interactive radiation (not homogenized in space), the aggregated cluster is indeed not always centered over the hot spot (Fig. 2b). In fact, once equilibrium is reached in the simulations with radiative feedbacks, the moist patch seems to decouple from the surface. It does not stay in the same location and can move across the domain. Thus this result that the convection is located over the hot spot is not robust once radiative feedbacks are accounted for. With radiative feedbacks, whether the 

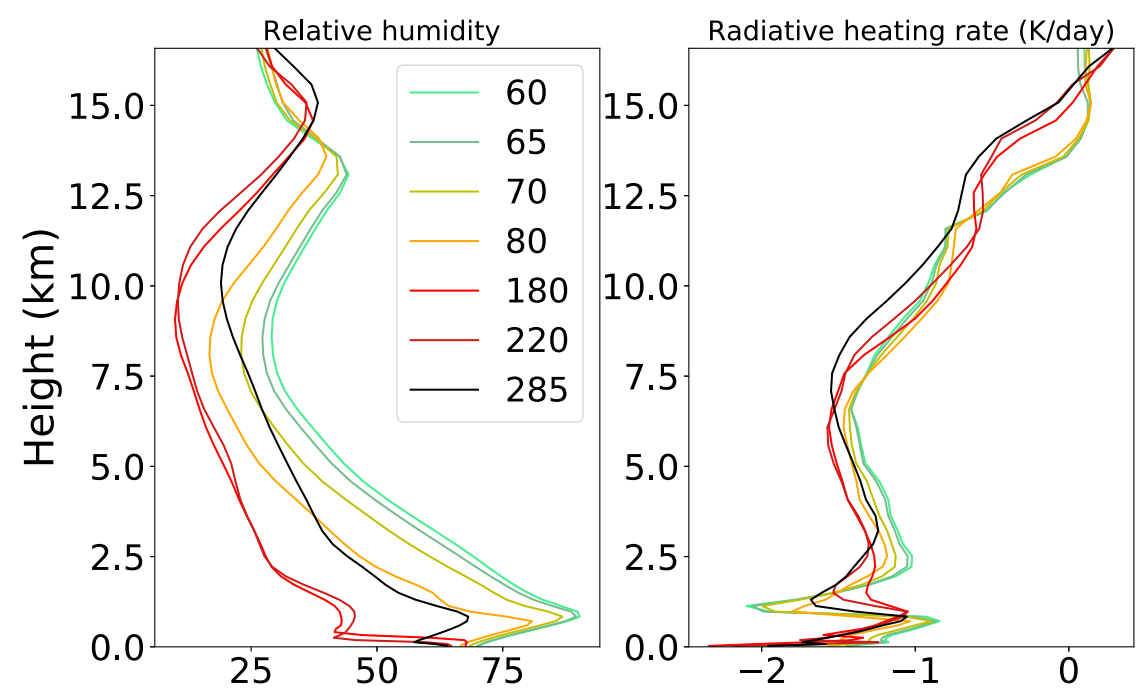

FIG. 10. Domain average vertical profiles averaged between day 35 and day 50 of the simulation for hot spots of different sizes. The domain average SST is $300 \mathrm{~K}$, the hot spot SST anomaly is $5 \mathrm{~K}$, and the domain size is $576 \times 576 \mathrm{~km}^{2}$.

convective cluster stays over the hot spot probably depends on the strength of radiative feedbacks compared to the hot spot effects.

Despite the large variability of the aggregation index and of the CRH pattern among the simulations without radiative feedbacks, maximum values of $W_{500}$ are always located over the hot spot (with an annular shape for $R \leq 65 \mathrm{~km}$ ) during the equilibrium phase (Fig. 9). A striking result is that the fractional area of large $W_{500}$ (e.g., $W_{500}>0.08 \mathrm{~m} \mathrm{~s}^{-1}$ ) is relatively independent of the radius of the hot spot. This region with large $W_{500}$ (Fig. 9b) has a fractional area of approximately $10 \%$ for all hot spot radii.

Figure 10 shows the vertical profiles of the domain mean relative humidity and radiative cooling rates at equilibrium. Simulations with large aggregation index have a drier average profile in agreement with low CRH in the dry patch (Fig. 6) and with earlier studies of self-aggregation. Average radiative cooling profiles are similar among the simulations which aggregate, with a large radiative cooling rate near the surface. These profiles are consistent with the very dry conditions and strong low-level radiative cooling accompanying aggregation found in earlier studies (Muller and Bony 2015).

\section{Conclusions}

In this paper, we investigate the role of persistent warm SST anomalies (hot spots) on the aggregation of deep convective clouds in cloud-resolving simulations. To this end, we perform simulations in radiative-convective equilibrium with SST anomalies of varying size and amplitude, but keeping the domain mean SST constant between simulations. Earlier studies with homogeneous SSTs find that radiative feedbacks are necessary for both the onset and maintenance of a self-aggregation of the convection for typical tropical temperatures $(\sim 300 \mathrm{~K})$. As for previous studies, we find that self-aggregation over homogeneous SSTs is favored at warm temperatures. We also find that the presence of a hot spot significantly accelerates the aggregation process and extends the range of average SSTs for which aggregation occurs.

We interpret these different behaviors by the fact that the mechanisms for convective aggregation with a hot spot or with homogeneous SSTs are different. With homogeneous SSTs, the aggregation of convection starts by a strengthening and an expansion of a dry region. Strong radiative cooling in dry regions yields enhanced subsidence that further dries the dry regions and that "pushes" low-level moisture toward the convective region (Fig. 7a). In other words, radiatively driven subsidence inhibits convection in the dry region (Wing et al. 2017; Bretherton et al. 2005; Muller and Held 2012).

With a hot spot, we find that aggregation (it is no more a selfaggregation since it is forced by the persistent SST anomaly) can occur even in the absence of radiative feedbacks (removed by homogenizing horizontally radiative cooling rates) if the hot spot is warm and large enough. The hot spot triggers aggregation by locally increasing the convective instability. Indeed, the warmer and moister conditions at low level over the hot spot favor deep convection, which brings the atmosphere toward a warmer condition. These warmer temperatures are imprinted over the whole domain through compensating subsidence warming in drier regions and via the propagation of gravity waves (Bretherton and Smolarkiewicz 1989). This subsidence favors further drying in dry regions. This is the positive feedback responsible for the expansion and strengthening of dry regions in hot spot simulations that aggregate. In other words, the hot spot "pulls" convection over itself, by generating a large-scale circulation with subsidence outside the hot spot (Fig. 7b).

In our simulations, planetary rotation is neglected so there is no limiting scale (beyond the dissipative scale) for the propagation of waves. So in our doubly periodic geometry, the subsidence compensates upward convective motion and is thus 
potentially stronger when the fractional area of the hot spot increases. In particular, for a given hot spot radius, the subsidence is sensitive to the domain size. This highlights the importance of using large domains when investigating island convection in similar nonrotating doubly periodic settings, in order to either avoid or control the triggering of selfaggregation feedbacks. In particular, the doubly periodic confinement of the large-scale circulation induced by surface heterogeneities may explain the nonmonotonic responses of precipitation to an island found in idealized simulations of convection over tropical islands. In such simulations, precipitation is found to increase and then decrease as a function of island radius holding the domain size fixed. Our results suggest that the large-scale circulation induced by the island may be impacted by the domain size if the domain is not large enough compared to the island.

In reality, with planetary rotation, the scale of the large-scale circulation induced by SST anomalies is likely determined by the Rossby radius of deformation. Our results suggest that for a large enough fractional area of SST anomalies compared to this large-scale circulation, self-aggregation feedbacks could play a role in organizing deep convection over SST anomalies. In the ocean, SST anomalies of the size studied here [O(10) km] are not uncommon, taking the form of mesoscale eddies (Chelton 2011). Their contribution to convective organization deserves further investigation. Finally, these findings raise questions on the organization of deep convection over tropical islands, e.g., of the Maritime Continent. There, a strong diurnal cycle further interacts with aggregation feedbacks and tendencies (Cronin et al. 2015). Our results show that the adjustment of the average temperature profile to the hot spot SST anomaly takes a few days for large hot spots, which is very slow compared to diurnal variability of surface temperature over tropical islands (reaching to a maximum typically in $6 \mathrm{~h}$ between sunrise and noon). Therefore, the atmosphere, and the convective aggregation pattern itself, will not have time to fully adjust before the island starts cooling down in the afternoon. Further work is needed to investigate the implication of our results on the diurnal cycle of convection over tropical islands.

Acknowledgments. This project has received funding from the Marie Sklodowska-Curie Actions (MSCA) under the European Union's Horizon 2020 Research and Innovation Programme (Grant Agreement 675675). The authors gratefully acknowledge funding from the French national program Les Enveloppes Fluides et l'Environnement (LEFE) of Institut National des Sciences de l'Univers (INSU), and from the program Paris Sciences et Lettres PSL-NYU (ANR-10-IDEX0001-02). Finally, the authors thank Kerry Emanuel and two anonymous reviewers for their useful comments about this work.

\section{REFERENCES}

Abbot, D. S., 2014: Resolved snowball Earth clouds. J. Climate, 27, 4391-4402, https://doi.org/10.1175/JCLI-D-13-00738.1.

Bellenger, H., J. Duvel, M. Lengaigne, and P. Levan, 2009: Impact of organized intraseasonal convective perturbations on the tropical circulation. Geophys. Res. Lett., 36, L16703, https:// doi.org/10.1029/2009GL039584.
Beringer, J., and N. Tapper, 2002: Surface energy exchanges and interactions with thunderstorms during the Maritime Continent Thunderstorm Experiment (MCTEX). J. Geophys. Res., 107, 4552, https://doi.org/10.1029/2001JD001431.

Bretherton, C. S., and P. K. Smolarkiewicz, 1989: Gravity waves, compensating subsidence and detrainment around cumulus clouds. J. Atmos. Sci., 46, 740-759, https://doi.org/10.1175/ 1520-0469(1989)046<0740:GWCSAD>2.0.CO;2.

—, P. N. Blossey, and M. Khairoutdinov, 2005: An energybalance analysis of deep convective self-aggregation above uniform SST. J. Atmos. Sci., 62, 4273-4292, https://doi.org/ 10.1175/JAS3614.1.

Chelton, D., 2011: Global observations of nonlinear mesoscale eddies. Prog. Oceanogr., 91, 167-216, https://doi.org/10.1016/ j.pocean.2011.01.002.

Collins, W. D., and Coauthors, 2006: The formulation and atmospheric simulation of the Community Atmosphere Model version 3 (CAM3). J. Climate, 19, 2144-2161, https://doi.org/ 10.1175/JCLI3760.1.

Coppin, D., and S. Bony, 2015: Physical mechanisms controlling the initiation of convective self-aggregation in a general circulation model. J. Adv. Model. Earth Syst., 7, 2060-2078, https://doi.org/10.1002/2015MS000571.

Cronin, T., K. A. Emanuel, and P. Molnar, 2015: Island precipitation enhancement and the diurnal cycle in radiative-convective equilibrium. Quart. J. Roy. Meteor. Soc., 141, 1017-1034, https:// doi.org/10.1002/qj.2443.

Crook, N. A., 2001: Understanding Hector: The dynamics of island thunderstorms. Mon. Wea. Rev., 129, 1550-1563, https://doi.org/ 10.1175/1520-0493(2001)129<1550:UHTDOI > 2.0.CO;2.

Daleu, C. L., R. S. Plant, and S. J. Woolnough, 2017: Using the weak-temperature gradient approximation to evaluate parameterizations: An example of the transition from suppressed to active convection. J. Adv. Model. Earth Syst., 9, 2350-2367, https://doi.org/10.1002/2017MS000940.

Emanuel, K. A., A. A. Wing, and E. M. Vincent, 2014: Radiativeconvective instability. J. Adv. Model. Earth Syst., 6, 75-90, https://doi.org/10.1002/2013MS000270.

Grabowski, W. W., 2006: Impact of explicit atmosphere-ocean coupling on MJO-like coherent structures in idealized aquaplanet simulations. J. Atmos. Sci., 63, 2289-2306, https:// doi.org/10.1175/JAS3740.1.

_- and M. W. Moncrieff, 2001: Large-scale organization of tropical convection in two-dimensional explicit numerical simulations. Quart. J. Roy. Meteor. Soc., 127, 445-468, https:// doi.org/10.1002/qj.49712757211.

Gray, W., and R. Jacobson, 1977: Diurnal variation of deep cumulus convection. Mon. Wea. Rev., 105, 1171-1188, https:// doi.org/10.1175/1520-0493(1977)105<1171:

DVODCC $>2.0 . \mathrm{CO} ; 2$.

Holloway, C. E., 2017: Convective aggregation in realistic convectivescale simulations. J. Adv. Model. Earth Syst., 9, 1450-1472, https:// doi.org/10.1002/2017MS000980.

Houze, R. A., Jr., 2004: Mesoscale convective systems. Rev. Geophys., 42, RG4003, https://doi.org/10.1029/2004RG000150.

Khairoutdinov, M. F., and D. A. Randall, 2003: Cloud resolving modeling of the ARM summer 1997 IOP: Model formulation, results, uncertainties, and sensitivities. J. Atmos. Sci., 60, 607-625, https://doi.org/10.1175/1520-0469(2003)060<0607: CRMOTA $>2.0 . \mathrm{CO} ; 2$.

, and K. A. Emanuel, 2010: Aggregated convection and the regulation of tropical climate. 29th Conf. on Hurricanes and Tropical Meteorology, Tucson, AZ, Amer. Meteor. Soc., P2.69, 
https://ams.confex.com/ams/29Hurricanes/techprogram/paper_ 168418.htm.

Kuang, Z., 2012: Weakly forced mock walker cells. J. Atmos. Sci., 69, 2759-2786, https://doi.org/10.1175/JAS-D-11-0307.1.

Mapes, B. E., 2001: Water's two height scales: The moist adiabat and the radiative troposphere. Quart. J. Roy. Meteor. Soc., 127, 2353-2366, https://doi.org/10.1002/qj.49712757708. , and R. Neale, 2011: Parameterizing convective organization to escape the entrainment dilemma. J. Adv. Model. Earth Syst., 3, M06004, https://doi.org/10.1029/2011MS000042.

Muller, C. J., 2013: Impact of convective organization on the response of tropical precipitation extremes to warming. J. Climate, 26, 5028-5043, https://doi.org/10.1175/JCLI-D-12-00655.1. , and P. A. O'Gorman, 2011: An energetic perspective on the regional response of precipitation to climate change. Nat. Climate Change, 1, 266-271, https://doi.org/10.1038/nclimate1169.

_ and I. M. Held, 2012: Detailed investigation of the self-aggregation of convection in cloud-resolving simulations. J. Atmos. Sci., 69, 2551-2565, https://doi.org/10.1175/JAS-D-11-0257.1.

- , and S. Bony, 2015: What favors convective aggregation and why? Geophys. Res. Lett., 42, 5626-5634, https://doi.org/ 10.1002/2015GL064260.

— , P. A. O'Gorman, and L. E. Back, 2011: Intensification of precipitation extremes with warming in a cloud-resolving model. J. Climate, 24, 2784-2800, https://doi.org/10.1175/2011JCLI3876.1.

Pauluis, O., and I. M. Held, 2002: Entropy budget of an atmosphere in radiative-convective equilibrium. Part I: Maximum work and frictional dissipation. J. Atmos. Sci., 59, 125-139, https://doi.org/ 10.1175/1520-0469(2002)059<0125:EBOAAI $>2.0$. CO;2.

Qian, J.-H., 2008: Why precipitation is mostly concentrated over islands in the Maritime Continent. J. Atmos. Sci., 65, 1428-1441, https://doi.org/10.1175/2007JAS2422.1.

Ramsay, H. A., and A. H. Sobel, 2011: Effects of relative and absolute sea surface temperature on tropical cyclone potential intensity using a single-column model. J. Climate, 24, 183-193, https://doi.org/10.1175/2010JCLI3690.1.

Romps, D. M., 2010: A direct measure of entrainment. J. Atmos. Sci., 67, 1908-1927, https://doi.org/10.1175/2010JAS3371.1.

Ruppert, J., and C. Hohenegger, 2018: Diurnal circulation adjustment and organized deep convection. J. Climate, 31, 4899-4916, https://doi.org/10.1175/JCLI-D-17-0693.1.

Sobel, A. H., and C. S. Bretherton, 2000: Modeling tropical precipitation in a single column. J. Climate, 13, 4378-4392, https:// doi.org/10.1175/1520-0442(2000)013<4378:MTPIAS >2.0.CO;2.

_ J. Nilsson, and L. M. Polvani, 2001: The weak temperature gradient approximation and balanced tropical moisture waves. J. Atmos. Sci., 58, 3650-3665, https://doi.org/10.1175/15200469(2001)058<3650:TWTGAA > 2.0.CO;2.
— C. D. Burleyson, and S. E. Yuter, 2011: Rain on small tropical islands. J. Geophys. Res., 116, D08102, https://doi.org/10.1029/ 2010JD014695.

Sugimoto, S., K. Aono, and S. Fukui, 2017: Local atmospheric response to warm mesoscale ocean eddies in the KuroshioOyashio confluence region. Sci. Rep., 7, 11871, https://doi.org/ 10.1038/s41598-017-12206-9.

Tan, J., C. Jakob, W. B. Rossow, and G. Tselioudis, 2015: Increases in tropical rainfall driven by changes in frequency of organized deep convection. Nature, 519, 451-454, https://doi.org/10.1038/ nature14339.

Tobin, I., S. Bony, and R. Roca, 2012: Observational evidence for relationships between the degree of aggregation of deep convection, water vapor, surface fluxes, and radiation. J. Climate, 25, 6885-6904, https://doi.org/10.1175/JCLI-D-11-00258.1.

Tompkins, A. M., 2001a: Organization of tropical convection in low vertical wind shears: The role of cold pools. J. Atmos. Sci., 58, 1650-1672, https://doi.org/10.1175/1520-0469(2001)058<1650: OOTCIL $>2.0 . \mathrm{CO} ; 2$.

- 2001b: Organization of tropical convection in low vertical wind shears: The role of water vapor. J. Atmos. Sci., 58, 529-545, https:// doi.org/10.1175/1520-0469(2001)058<0529:OOTCIL >2.0.CO;2.

— , and G. C. Craig, 1998: Radiative-convective equilibrium in a three-dimensional cloud-ensemble model. Quart. J. Roy. Meteor. Soc., 124, 2073-2097, https://doi.org/10.1002/qj.49712455013.

Wang, S., and A. H. Sobel, 2011: Response of convection to relative sea surface temperature: Cloud-resolving simulations in two and three dimensions. J. Geophys. Res., 116, D11119, https:// doi.org/10.1029/2010JD015347.

- , and — 2017: Factors controlling rain on small tropical islands: Diurnal cycle, large-scale wind speed, and topography. J. Atmos. Sci., 74, 3515-3532, https://doi.org/10.1175/JAS-D16-0344.1.

Wing, A. A., and K. A. Emanuel, 2014: Physical mechanisms controlling self-aggregation of convection in idealized numerical modeling simulations. J. Adv. Model. Earth Syst., 6, 59-74, https://doi.org/10.1002/2013MS000269.

— long channel geometry. Quart. J. Roy. Meteor. Soc., 142, 1-15, https://doi.org/10.1002/qj.2628.

— K. Kmanuel, C. Holloway, and C. Muller, 2017: Convective self-aggregation in numerical simulations: A review. Surv. Geophys., 38, 1173-1197, https://doi.org/10.1007/s10712-0179408-4.

Zuidema, P., G. Torri, and C. Muller, 2017: Precipitation-induced atmospheric cold pools over oceans and their interactions with the larger-scale environment. Surv. Geophys., 38, 1283-1305, https://doi.org/10.1007/s10712-017-9447-x. 